\title{
Development of Alkali-silica-reaction Suppressing Material with Li-containing Zeolite
}

\author{
Motoki UEHARA, Dr. Eng. \\ Senior Researcher, \\ Kiyoshi MIZUNO \\ Senior Researcher, \\ Takatsune SATO \\ Researcher, \\ Concrete Materials Laboratory, Materials Technology Division
}

\begin{abstract}
The alkali-silica-reaction (ASR) suppression effect of Li-containing zeolite prepared from metakaolin was found to be greater than that of commercial Ca-A type zeolite. Material containing more Li-ABW type zeolite suppressed ASR more effectively when used as an admixture. In contrast, material containing more Li-EDI type zeolite was more effective in suppressing ASR when employed as grout for cracks. Trial samples were made of a new crack grout with Li-EDI type zeolite, which is more effective in suppressing ASR than a commercial crack grout with Ca-A type zeolite. No problems were detected with the physical characteristics of this material when used as grout.
\end{abstract}

Keywords: Li-EDI type zeolite, Li-ABW type zeolite, metakaolin, alkali-silica-reaction

\section{Introduction}

Water-soluble alkali components such as potassium ions $\left(\mathrm{K}^{+}\right)$or sodium ions $\left(\mathrm{Na}^{+}\right)$contained in cement or supplied from external sources such as seawater or anti-freezing agents cause increases in the $\mathrm{pH}$ values of micropores in concrete. The resulting alkaline conditions can lead to reactions with certain aggregate components in concrete, forming a gel that absorbs water and results in swelling. This is known as an alkali-silica-reaction (hereinafter referred to as ASR). The gel form through ASR absorbs water and expands, which often leads to cracking in concrete (Fig. $1)$.

Among the various methods adopted to repair concrete affected by ASR is the use of calcium (Ca)-A-type zeolite (hereinafter referred to as Ca-A) [1]. With this method, ultra-fine cement containing $\mathrm{Ca}-\mathrm{A}$ is injected under low pressure into concrete cracks as shown in Fig. 1, and a water-repellent water-based silane impregnate material is then placed over the surface of the concrete. However, con-

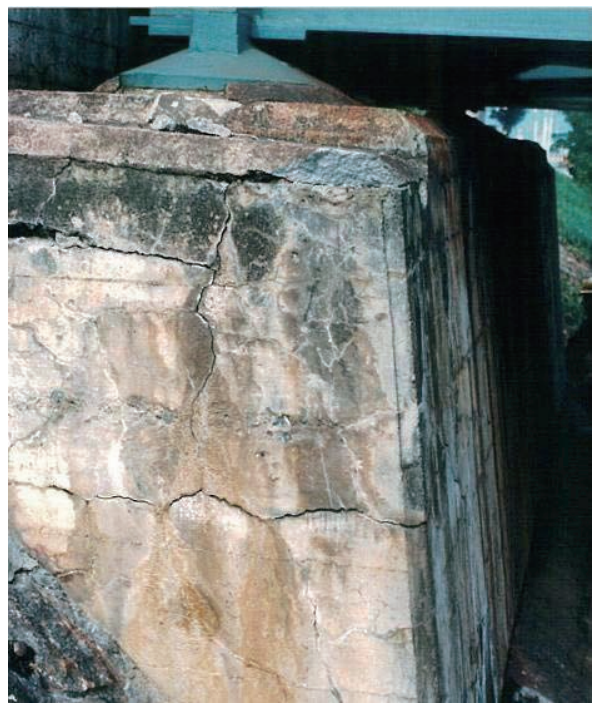

Fig. 1 Structure affected by ASR crete repaired in this way is often affected by ongoing ASR, which provokes deformation again within 10 years or so. Against this background, there is demand for a material which can provide a longer-lasting suppression effect.

Recently, lithium ( $\mathrm{Li}$ ) ions were identified as owning ASR suppression properties, leading to the development of a repair method in which a $\mathrm{Li}$ nitrite solution is pressfitted into concrete [2]. However, the technique requires large amounts of Li solution, and press-fitting is timeconsuming. Accordingly, a number of issues remain to be resolved with this approach.

The authors focused on the use of $\mathrm{Li}$ ions and zeolite to clarify the effectiveness of Li-A-type zeolite (hereinafter referred to as Li-A) in suppressing ASR [3]. However, as this method requires repeated cycles of ion exchange for synthesis, large amounts of Li compound are required, which creates a corresponding increase in cost. Elsewhere, Matsumoto et al. succeeded in directly synthesizing LiEDI-type zeolite (hereinafter referred to as Li-EDI) and Li-ABW-type zeolite (hereinafter referred to as Li-ABW) as materials that do not need any ion exchange process to pass through [4]. Li-EDI and Li-ABW both have silicon ( $\mathrm{Si}$ ) to aluminum $(\mathrm{Al})$ ratios of 1 in their respective frameworks - values which are identical to that of Li-A. They therefore have the same $\mathrm{Li}$ content as $\mathrm{Li}-\mathrm{A}$, and are therefore expected to exhibit significant ASR suppression effects. The method of Matsumoto et al., in which reagents are used as starting materials, inevitably involves high costs. Accordingly, the authors developed a method in which low-priced metakaolin (an amorphous substance formed by burning the clay mineral kaolin), which is used as an admixture for concrete, is used to synthesize the above zeolites. The results produced the following findings [5]:

(1) Metakaolin can be mixed with the minimum necessary amount of lithium hydroxide $(\mathrm{LiOH})$ solution to create a suspension, which can then be made to react at $20-90{ }^{\circ} \mathrm{C}$ and synthesize a substance containing Li-EDI and Li-ABW.

(2) Reactions can be carried out at lower or higher temperatures to provide a substance with higher content of Li-EDI or Li-ABW, respectively. 
Table 1 Outline of the samples used for each examination

\begin{tabular}{|c|c|c|c|c|}
\hline & & Li-EDI a & Li-ABW & \\
\hline & Sample name & rati & $(\%)$ & Notes \\
\hline & & Li-EDI & Li-A BW & \\
\hline (1) & $1 \mathrm{M} 20(168 \mathrm{~h})$ & 61.2 & 0.0 & \\
\hline (2) & $1 \mathrm{M} 40$ (48 h) & 56.0 & 16.2 & \\
\hline (3) & $1 \mathrm{M} 60$ (48 h) & 8.9 & 47.9 & A morphous materials with a zeolite-like \\
\hline (4) & $1 \mathrm{M} 90(5 \mathrm{~h})$ & 0.0 & 60.8 & \\
\hline (5) & $1 \mathrm{M} 90$ (48 h) & 0.0 & 63.5 & \\
\hline (6) & $4 \mathrm{M} 40$ (48 h) & 0.0 & 0.0 & M ain substances: amorphous materials \\
\hline (7) & 4M 60 (48 h) & 0.0 & 11.7 & with a zeolite-like structure \\
\hline (8) & Li-ABW & 0.0 & 100 & Single nhase camnle of each zeolite \\
\hline (9) & Li-EDI & 100 & 0.0 & 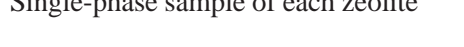 \\
\hline (10) & Li-A & $-\cdots--$ & $-\cdots$ & Single-phase sample of Li-A \\
\hline (11) & Ca-A & 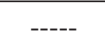 & 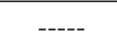 & Single-phase sample of Ca-A \\
\hline The & on of (1) - (7) & espond & the sam & le names employed by U ehara et al [5]. \\
\hline Exan & $1 \mathrm{M} 90(5 \mathrm{~h}) \mathrm{re}$ & esents a & Inthesis tin & e of 5 hours, a synthesis temperature of \\
\hline $90{ }^{\circ} \mathrm{C}$, a & 1M-concentro & ion LiOH & olution. & \\
\hline material - & o each contai & d small & nounts 0 & nica and quartz (present in the starting \\
\hline
\end{tabular}

(3) A high-concentration $\mathrm{LiOH}$ solution can be used to produce an amorphous substance structurally similar to zeolite.

This article details the ASR suppression effects of LiEDI, Li-ABW and the amorphous substance structurally similar to zeolite, as prepared using the above method. The substances were used to obtain individual batches of crack grout, whose properties were then studied. The results are reported below.

\section{Preparation of Li-containing Zeolite}

The Li-containing zeolites were prepared according to the method described by Uehara et al. [5]. The Li-containing zeolites employed are listed in Table 1, where (1) to (7) show those synthesized from metakaolin using the Uehara et al. [5] method, (8) and (9) show those synthesized from reagents using the Matsumoto et al. [4] method and (10) shows the Li-A synthesized from ion exchange processes using the Uehara [3] method The Ca-A shown for (11) is commercial zeolite made by Nippon Chemical Industrial Co. Ltd.

\section{Test to investigate ASR suppression effects with products intended for use as admixtures}

\subsection{Outline of examination}

The synthesized products shown in Table 1 (intended for use as admixtures for concrete) were added when the mortar was prepared, and were tested to clarify their ASR suppression effects. Table 2 shows the compounding conditions adopted in mortar preparation. The amounts of synthesized product to be added were controlled so that the $\mathrm{Li}$ amounts were equal to that of $1 \mathrm{M} 40(48 \mathrm{~h})$, which was defined as the standard (i.e., 10 mass \%). The amount of Ca-A added was 10 mass\%. Mortar expansion was measured in line with JIS (Japanese Industrial Standards) A 1146 Test method for alkali-silica-reactivity of aggregates by mortarbar method.

\subsection{Results}

Figure 2 shows the results of testing to investigate accelerated expansion of mortar prepared with the compounding shown in Table 2. The Li-ABW synthesized from the reagents visibly produced the mortar with the lowest expansion rate. The $1 \mathrm{M}$ samples synthesized with a relatively low treatment solution concentration and high amounts of produced zeolite show a trend of lower expansion with higher Li-ABW content. Low expansion rates are also seen for the $4 \mathrm{M}$ samples synthesized with a high

Table 2 Mixing proportions of the mortar with added zeolite

\begin{tabular}{ccccc}
\hline \multirow{2}{*}{ Sample name } & $\begin{array}{c}\text { N cement } \\
(\mathrm{g})\end{array}$ & $\begin{array}{c}\text { A mount added } \\
(\mathrm{g})\end{array}$ & $\begin{array}{c}\text { Reactive aggregate } \\
(\mathrm{g})\end{array}$ & $\begin{array}{c}\text { W ater } \\
(\mathrm{g})\end{array}$ \\
\hline N o addition & 600.0 & 0.0 & 1,350 & 300 \\
1M 40 (48 h) & 540.0 & 60.0 & 1,350 & 330 \\
1M 60 (48 h) & 542.2 & 57.8 & 1,350 & 330 \\
1M 90 (48 h) & 573.4 & 53.2 & 1,350 & 330 \\
4M 40 (48 h) & 573.4 & 53.2 & 1,350 & 330 \\
4M 60 (48 h) & 576.7 & 46.6 & 1,350 & 330 \\
Li-A B W & 575.6 & 48.8 & 1,350 & 330 \\
Li-EDI & 570.0 & 60.0 & 1,350 & 330 \\
Li-A & 571.1 & 57.8 & 1,350 & 330 \\
Ca-A & 570.0 & 60.0 & 1,350 & 330 \\
1M 20 (168 h) & 534.2 & 65.8 & 1,350 & 311 \\
1M 90 (5 h) & 549.6 & 50.4 & 1,350 & 311 \\
\hline
\end{tabular}

Alkali amount $\left(\mathrm{R}_{2} \mathrm{O}\right): 2.8$ mass $\%$ of the cement 


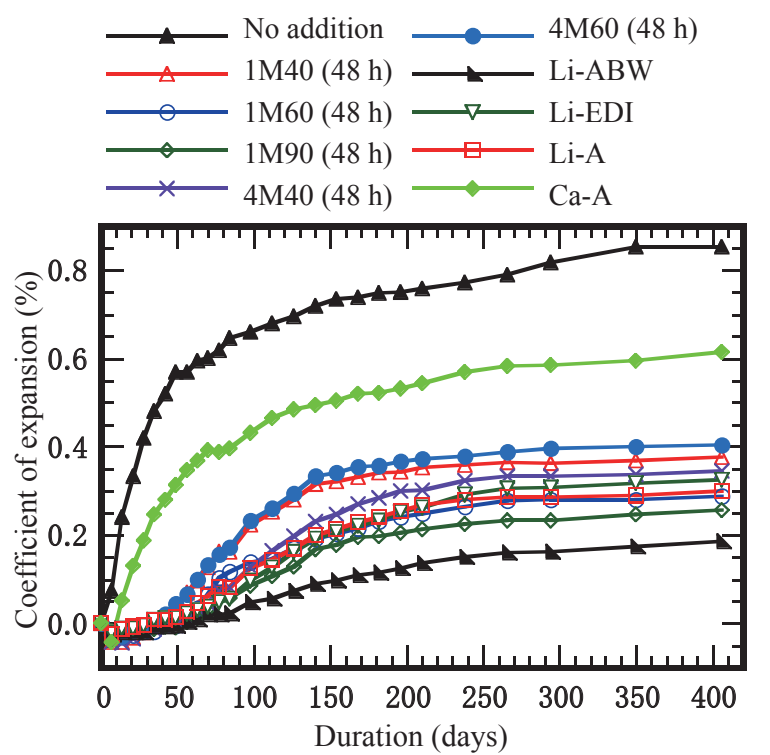

Fig. 2 Coefficients of expansion for product-added mortar types

Amount of alkali: $2.8 \%$

Curing temperature: $40{ }^{\circ} \mathrm{C}$

treatment solution concentration and negligible amounts of produced zeolite, and an ASR suppression effect can therefore be expected. This is conceivable since the $4 \mathrm{M}$ samples included Li-containing amorphous aluminosilicate substances structurally similar to zeolite, thereby creating ion exchange capability, even though they contained no crystalline zeolite. These amorphous substances are expected to be utilized in the future, but currently involve prohibitively high material costs because any surplus Li solution is wasted. In order for the current synthesized products to be usable as ASR suppression materials, it is preferable to select the 1M90 series of samples, which have high LiABW content and significant ASR suppression effects, and involve less wastage of $\mathrm{Li}$ solution.

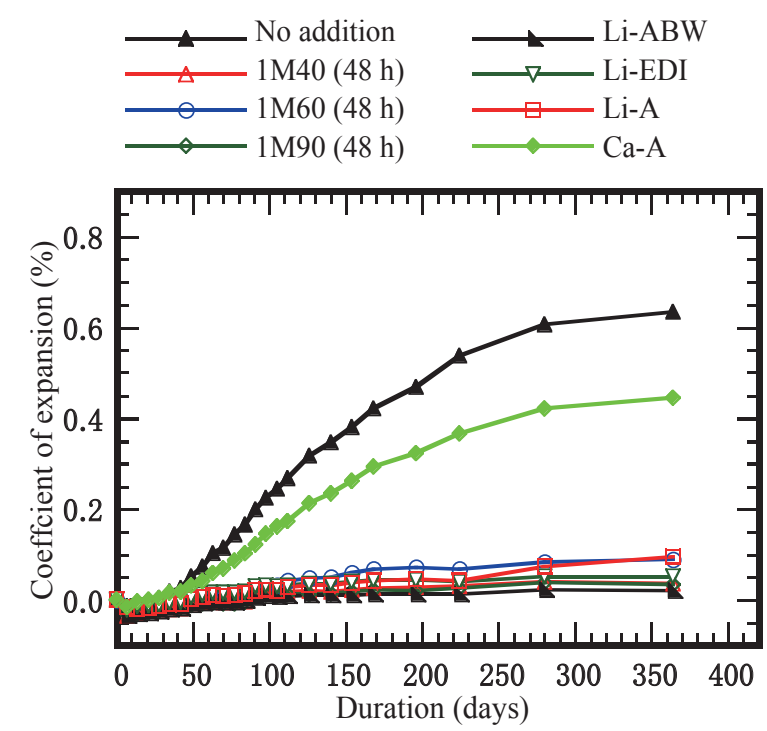

Fig. 3 Coefficients of expansion for product-added mortar types

Amount of alkali: $2.8 \%$

Curing temperature: $20^{\circ} \mathrm{C}$

Figure 3 shows the results of testing to investigate the expansion of mortar cured at $20{ }^{\circ} \mathrm{C}$ - a temperature similar to that found in practical working conditions. It presents the same trends as those seen for expansion in concrete with accelerated curing, indicating that the samples with high Li-ABW content showed low expansion rates and large ASR suppression effects.

Table 3 Mixing proportions of the mortar for the injection test $\left(\mathrm{R}_{2} \mathrm{O}=\mathbf{1 . 6} \%\right)$

\begin{tabular}{cccc}
\hline N P cement $(\mathrm{g})$ & A ggregate $(\mathrm{g})$ & Water $(\mathrm{g})$ & $\mathrm{N} \mathrm{aOH}(\mathrm{g})$ \\
\hline 600.0 & 1350.0 & 300.0 & 7.9 \\
\hline \multicolumn{4}{c}{ A ggregate: A SR-reactive andesite }
\end{tabular}

Table 4 Mixing proportions of the mortar with added zeolite

\begin{tabular}{|c|c|c|c|c|c|}
\hline $\begin{array}{l}\text { Sample } \\
\text { name }\end{array}$ & $\begin{array}{l}\text { B ase paste } \\
\text { species }\end{array}$ & $\begin{array}{l}\text { A mount of base } \\
\text { paste }(\mathrm{g})\end{array}$ & $\begin{array}{c}\text { A mount of product } \\
\text { added }(\mathrm{g})\end{array}$ & $\begin{array}{l}\text { A mount of liquid } \\
\text { mixture }(\mathrm{g})\end{array}$ & $\begin{array}{c}\text { A mount of } \\
\text { water }(\mathrm{g})\end{array}$ \\
\hline \multirow{2}{*}{ No addition } & B B & 400 & 0 & --.-- & 120.0 \\
\hline & Armor & 300 & 0 & 240 & --- \\
\hline \multirow{3}{*}{ 1M 20 (168 h) } & $B B$ & 180 & 120 & ----- & 173.3 \\
\hline & B B & 320 & 80 & ---- & 187.8 \\
\hline & Armor & 240 & 60 & 240 & --- \\
\hline \multirow{3}{*}{$1 \mathrm{M} 90$ (5 h) } & B B & 180 & 120 & ---- & 164.8 \\
\hline & $B B$ & 320 & 80 & ---- & 165.6 \\
\hline & Armor & 240 & 60 & 240 & $-\cdot-$ \\
\hline \multirow{3}{*}{ Ca-A } & B B & 180 & 120 & --- & 94.6 \\
\hline & $B B$ & 320 & 80 & --- & 125.2 \\
\hline & Armor & 240 & 60 & 240 & --- \\
\hline \multirow{3}{*}{ Li-A } & $B B$ & 180 & 120 & --- & 117.6 \\
\hline & $B B$ & 320 & 80 & --- & 129.8 \\
\hline & Armor & 240 & 60 & 240 & --- \\
\hline
\end{tabular}

BB: B-species blast-furnace slag cement

A rmor: A rmor \#600P cracked grout (M itsubishi Materials Corporation) 


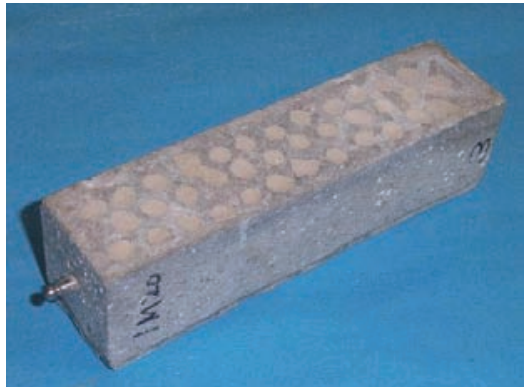

Fig. 4 Photo of mortar in the injection construction test

4. Test to investigate ASR suppression effects with products intended for use as crack grout

\subsection{Outline of examination}

When the synthesized products shown in Table 1 are used as crack grout, the Li ions inserted into cracks must permeate the concrete and adsorb alkali ions in order to be effective. In this test, holes were drilled into mortar samples considered to be vulnerable to the effects of ASR, and were filled using cement paste with Li-containing zeolites (Fig.4). The resulting ASR suppression effects were studied in line with JIS A 1146. Table 3 shows the test mortar sample compositions, and Table 4 shows those of the crack grout.

\subsection{Results}

Figures 5 to 7 show the results of expansion measurements on the injected test samples. In contrast to the results shown in Figs. 2 and 3, the mortar sample injected with the $1 \mathrm{M} 20$ paste $(168 \mathrm{~h})$ mainly containing Li-EDI showed less expansion and a greater suppression effect than the sample injected with $1 \mathrm{M} 90(5 \mathrm{~h})$ paste mainly containing Li-ABW. Figures 6 and 7 show the expansion rate difference between the pastes containing only $20 \%$ of the commercial Ca-A or 1M20 (168 h) compounds was larger than the difference between the pastes with a $40 \%$ content. Even a small 1M20 (168 h) content resulted in low expansion of the mortar test sample, suggesting that Li-EDI for crack grout exhibits a significant ASR suppression effect.

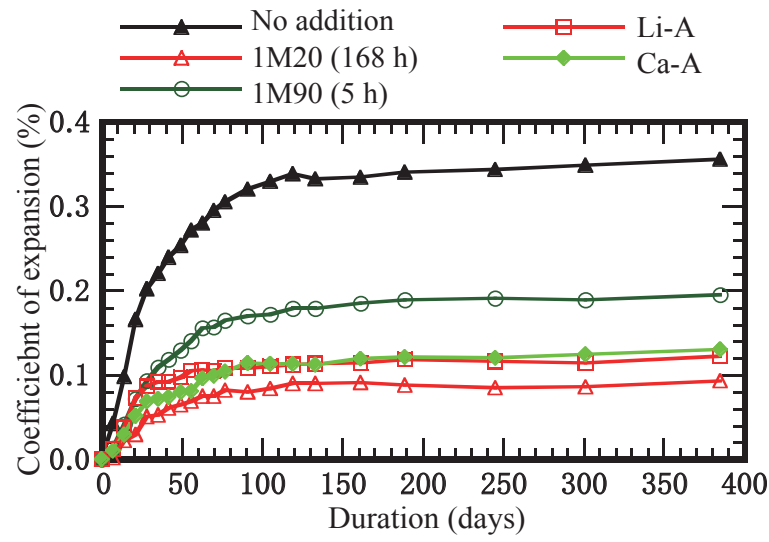

Fig. 5 Coefficients of expansion for mortar types injected with $\mathbf{4 0}$ mass\% zeolite-added BB cement paste

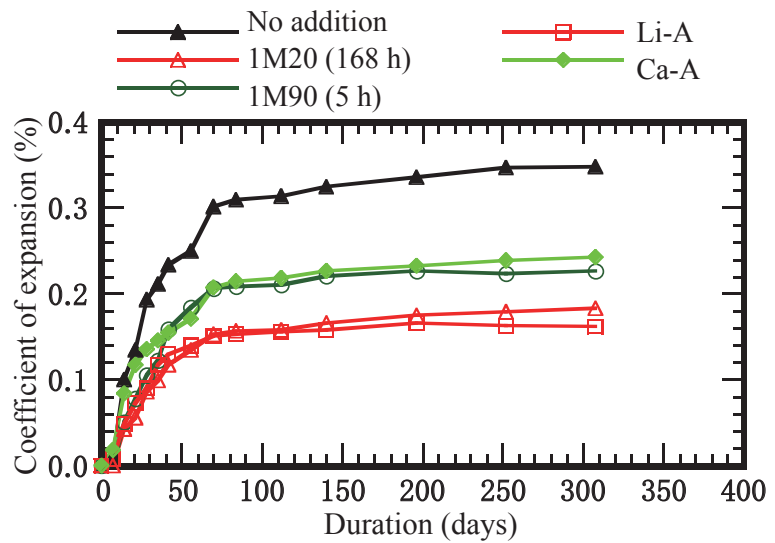

Fig. 6 Coefficients of expansion for mortar types injected with 20 mass \% zeolite-added BB cement paste

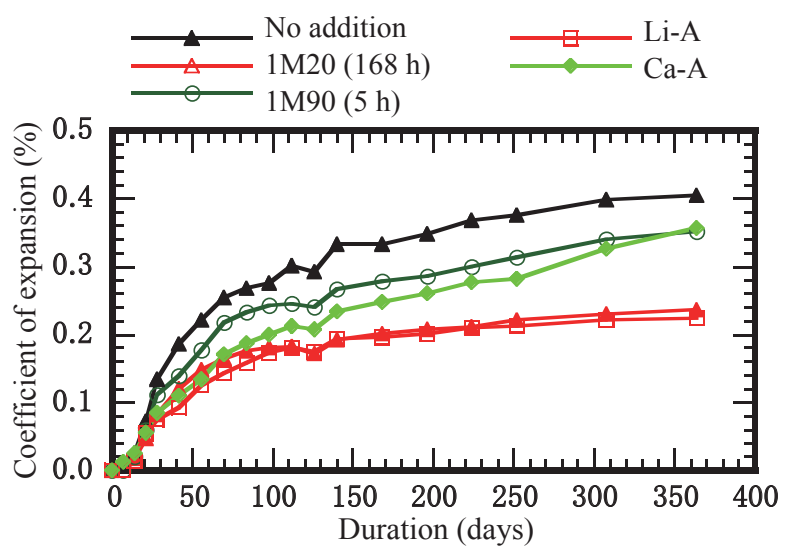

Fig. 7 Coefficients of expansion for mortar types injected with 20 mass\% zeolite-added commercial grout (Armor \#600P, Mitsubishi Materials Corporation)

5. Test to investigate the ASR suppression mechanism

\subsection{Outline of examination}

In this test, the release of $\mathrm{Li}^{+}$and the adsorption of alkali ions in cement paste were measured to clarify the ASR suppression mechanism of synthesized Li-containing zeolite. The experimental processes are outlined below.

To make the cement paste, precise amounts of the synthesized products shown in Table 1 were added to ordinary Portland cement to ensure uniform Li content. After a fixed period of time, the paste was crushed, and $1 \mathrm{~g}$ of the resulting mass was added to $20 \mathrm{ml}$ of water at $30{ }^{\circ} \mathrm{C}$ and stirred for three hours to elute the water-soluble $\mathrm{Li}, \mathrm{Na}$ and $\mathrm{K}$ in the concrete's fine pores into the solution, which was then quantitatively analyzed using an atomic absorption spectrometer to quantify the amount of Li released. Cement paste with no synthesized product was similarly treated to determine its $\mathrm{Na}$ and $\mathrm{K}$ content, and the amount of $\mathrm{Na}$ and $\mathrm{K}$ found in the cement paste containing the synthesized product was subtracted from these values. The result was defined as the amount of $\mathrm{Na}$ and $\mathrm{K}$ adsorbed by the synthesized product. 


\subsection{Results}

The results showed that the Li-ABW-rich sample used as an admixture and the Li-EDI-rich sample used for crack grout each exhibited significant ASR suppression effects. The ASR suppression mechanism behind these Li-containing zeolites was therefore investigated.

Figures 8 and 9 show the amounts of Li released from the synthesized product and the amounts of $\mathrm{Na}^{+}$and $\mathrm{K}^{+}$ immobilized in cement paste containing the product over time. The amount of $\mathrm{Li}$ released was calculated by taking the amount required to occupy all $\mathrm{Li}$ sites inside the crystalline structure of the synthesized product as $100 \%$. The immobilization rate was also expressed as the ratio of the total amount of $\mathrm{Na}$ and $\mathrm{K}$ adsorbed to the amount of $\mathrm{Li}$ released.

Li-EDI synthesized from the reagents released larger amounts of $\mathrm{Li}$ from the early stage than Li-ABW. It was considered that Li-EDI, which is less stable in alkaline conditions than Li-ABW, was liable to break down structurally in the cement paste. Additionally, Li-EDI and Ca-A had a higher capacity to immobilize $\mathrm{Na}$ and $\mathrm{K}$ (Fig. 9) than Li-ABW because the latter has lower ion exchange performance in terms of selecting $\mathrm{Na}$ and $\mathrm{K}$. However, it was considered that most zeolites broke down in the early stages and that the remaining zeolites immobilized $\mathrm{Na}$ and $\mathrm{k}$ ions because the immobilization rate was lower than the $\mathrm{Li}$ release rate. The sample synthesized from metakaolin at a lower temperature to contain large amounts of Li-EDI (here, sample 1M40 (48 h)) also released large amounts of $\mathrm{Li}$ in the early stage, creating a significant alkali immobilization effect. Amorphous samples such as those of $4 \mathrm{M} 40$ and $4 \mathrm{M} 60$ also released $\mathrm{Li}$ ions and adsorbed $\mathrm{Na}$ and $\mathrm{K}$ ions in the same way as zeolite.

As seen from the above results, Li-EDI had a significant ASR suppression effect in crack grout because it caused extensive $\mathrm{Li}$ ion release, which immobilized alkali ions around the crack and suppressed ASR expansion in the peripheral area. Li-ABW blended as an admixture also exhibited a significant ASR suppression effect, but the reason for this is unclear. One possible reason is that using it as an admixture had physical effects including densification of the cement paste in addition to the above chemical factors, which influenced the ASR suppression effect.

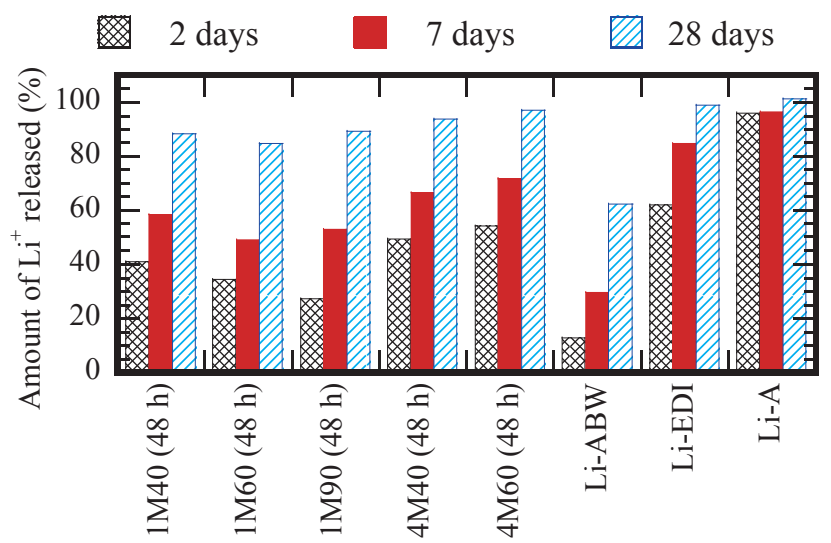

Fig. 8 Amount of $\mathrm{Li}^{+}$released from products in cement paste

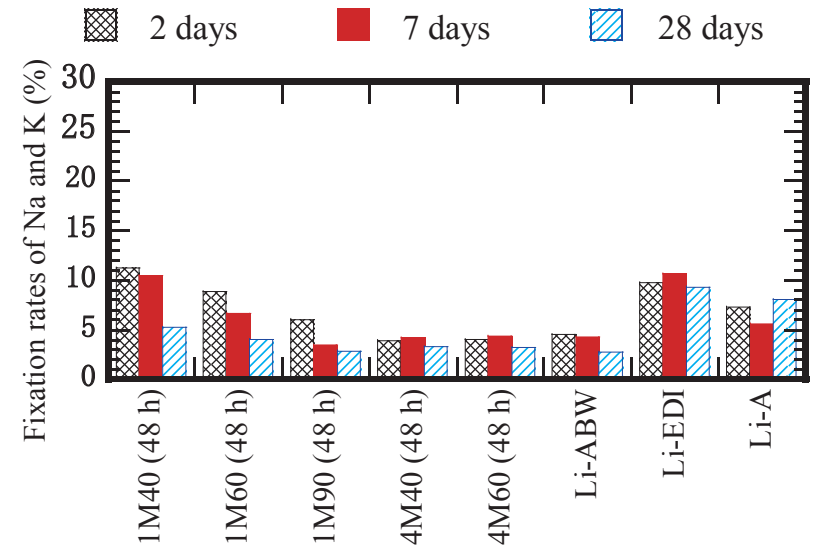

Fig. 9 Fixation rates of $\mathrm{Na}$ and $\mathrm{K}$ ions against products in cement paste

\section{Preparation and property testing of crack grout}

\subsection{Preparation}

The Ca-A component in conventional Ca-A-containing cement base grout was substituted with Li-EDI-rich 1M20 $(168 \mathrm{~h})$ to prepare a cement base crack grout with Li-containing zeolite. The trial grout was prepared with a blend amount of $2.5 \mathrm{~kg}$ per batch, water was supplied in the standard amount of $68 \%$, and a water-reducing material was supplied to adjust freshness. The materials were blended using a hand mixer for $2 \mathrm{~min}$.

\subsection{Property testing}

\subsubsection{Outline of examination}

A freshness test on the crack grout prepared was carried out in a room with an atmospheric temperature of 20 $\pm 2{ }^{\circ} \mathrm{C}$ with humidity of $50 \%$ or more using the method shown in Table 5. Tests were performed to determine the properties after curing in line with JSCE (Japan Society of Civil Engineers)-K542-2010, Test methods for cement crack injection materials for repairs on concrete structures (proposal).

\subsubsection{Results}

The Li-EDI-rich cement base crack grout with 1M20 (168 h) and a high ASR suppression effect was tested for various capabilities. To allow workability and crack-filling capability, grout must be fresh with good fluidity (i.e., it must filter quickly), a moderately long pot life (about 10 hours before onset of coagulation), and minimal temporal change in fluidity properties such as the flow-down time and viscosity. In addition to ensure that cracks remain completely filled after curing, it must have curing properties that result in sufficient strength, sufficient adhesiveness to the concrete, and moderate water absorption.

Tables 6 and 7 show the results of testing in relation to freshness and properties after curing of the crack grout prepared and commercial crack grout for ASR. It can be seen that the Li-EDI-rich crack grout with 1M20 (168 h) used in the test showed a tendency to develop somewhat 
Table 5 Test methods for the fresh properties of cracking grout

\begin{tabular}{ll} 
P funnel flow time & JSCE-F521-1999 \\
JA funnel flow time & JSCE-F531-1999 \\
Viscosity & M easured using a B-type rotational viscometer \\
Mass of unit volume & JIS A 1116 \\
Setting time & Measured using an automated coagulation testing machine \\
\hline
\end{tabular}

Table 6 Results of the fresh properties test for the grout prototype

\begin{tabular}{|c|c|c|c|}
\hline \multicolumn{2}{|l|}{ Type of grout } & A rmor \#610* & 1M 20 (168 h) - substituted sample e $^{* *}$ \\
\hline \multicolumn{2}{|c|}{ Temperature of mixed grout $\left({ }^{\circ} \mathrm{C}\right)$} & 21.8 & 22.3 \\
\hline \multirow{2}{*}{ P funnel flow time (s) } & Immediately & 10.0 & 10.3 \\
\hline & A fter 60 minutes & 10.0 & 10.8 \\
\hline JA funnel flow time (s) & Immediately & 12.5 & 13.1 \\
\hline \multirow{2}{*}{ V iscosity $(\mathrm{mPa} \cdot \mathrm{s})$} & Immediately & 34 & 20 \\
\hline & A fter 60 minutes & 56 & 20 \\
\hline$M$ ass of unit volume $\left(t / m^{3}\right)$ & & 1.62 & 1.62 \\
\hline \multirow[t]{2}{*}{ Setting time (hours-minutes) } & Initial setting time & $15-50$ & $10-20$ \\
\hline & Final setting time & $20-10$ & $16-30$ \\
\hline
\end{tabular}

Table 7 Results of the properties test for the hardened grout prototype

\begin{tabular}{lcc}
\hline & $\begin{array}{c}\text { Armor } \\
\# 610^{*}\end{array}$ & $\begin{array}{c}1 \mathrm{M} 20(168 \mathrm{~h})- \\
\text { substituted sample** }\end{array}$ \\
\hline W ater retention coefficient (\%) & $27^{+}$ & $27^{+}$ \\
Compressive strength $\left(\mathrm{N} / \mathrm{mm}^{2}\right)$ & 14.0 & $13.9-11.1$ \\
M odulus of static elasticity $\left(\mathrm{N} / \mathrm{mm}^{2}\right)$ & 7,890 & $9,180-9,210$ \\
Flexural strength $\left(\mathrm{N} / \mathrm{mm}^{2}\right)$ & 5.1 & $5.8-4.9$ \\
B onding strength $\left(\mathrm{N} / \mathrm{mm}^{2}\right)$ & 2.7 & $3.3-3.4$ \\
Coefficient of water absorption $(\%)$ & 30.4 & $27.9-25.7$
\end{tabular}

$*$ A mount of plasticizer $=1.0 \%$ ** A mount of plasticizer $=1.0 \%-1.5 \%$

poor fluidity in comparison with the commercial crack grout for ASR, however, this could be slightly adjusted using a water-reducing material to meet the fundamental capability requirements.

\section{Crack injection test}

\subsection{Outline of examination}

A columnar test subject and a large-scale test subject with cracks were injected with the crack grout preparation.

\subsubsection{Columnar test subject}

The concrete columnar test subject $(\phi 10 \times 20 \mathrm{~cm})$ was circumferentially covered with epoxy resin to bind it, and was then cut to simulate cracking. The cracks were filled with grout using a low-pressure injection tool to enable observation of the injection characteristics.

\subsubsection{Large-scale concrete test subject}

Cracks with a width of $0.2 \mathrm{~mm}$ or more in the largescale concrete test subject $(50 \times 50 \times 100 \mathrm{~cm})$ affected by ASR-related cracking were injected with grout under low pressure.

\subsection{Results}

\subsubsection{Columnar test subject}

Figure 10 shows simulated cracks in the columnar test subject injected with crack grout. Even the finest cracks are sufficiently filled; none of the problem areas are seen.

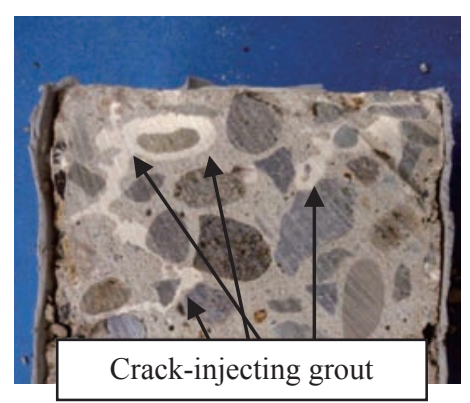

Fig. 10 Grout injected into the simulated cracks

\subsubsection{Large-scale concrete test subject}

Figure 11 shows the work performed to inject grout with Li-EDI (1M20 (168 h)) into ASR-related cracks in the large-scale concrete test subject $(50 \times 50 \times 100 \mathrm{~cm})$ under low pressure. No workability problems were found indicating that the developed material could be employed as 

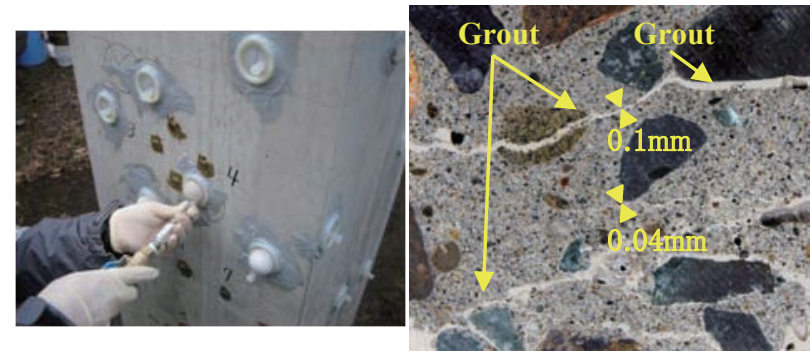

Fig. 11 Grout injection for a large test specimen, and an enlarged photo of the grout injected into the crack

a direct substitute for conventional crack grout. The photograph on the right of Fig. 11 shows a magnification of grout-injected cracks, and indicates that cracks as small as $0.04 \mathrm{~mm}$ in width could be filled. It is therefore concluded that the trial grout is suitable for injection into cracks caused by ASR.

\section{Conclusions}

Metakaolin was utilized to prepare samples combining $\mathrm{Li}$ ions with zeolite, each of which was individually known to significantly suppress ASR. These samples were then studied to determine the relative ASR suppression effects. Results demonstrated that using a Li-ABW-rich sample of a material used as an admixture added during the early stage of concrete manufacture exhibited a large ASR suppression effect. The Li-EDI-rich sample used for crack grout also demonstrated a similarly significant effect.
The Li-EDI-rich sample prepared from metakaolin was used as a substitute for Ca-A, which is the main component of a conventional ASR-suppressing inorganic base crack grout, to create a new grout that was then tested to determine its crack-filling capability. The results showed that even the finest cracks with a width of approximately $0.04 \mathrm{~mm}$ could be effectively filled with the grout. It was therefore concluded that the trial grout could be practically usable to treat cracks.

\section{References}

[1] Domon K., Moriya S., Masuda Y. Taki T., “An Experimental Study on AAR-Inhibiting Effects of Various Repair Methods," Proceedings of the Japan Concrete Institute, Vol.23, pp.409-414, 2001 (in Japanese).

[2] Era K., Mihara T., Okada S., Miyagawa T., "Control of Alkali-Silica Expansion by Lithium Ion Pressurized Injection Method," Journal of the Society of Material Science, Japan, Vol.57, No.10, pp.993-998, 2008 (in Japanese).

[3] Uehara M., "Restraint of Alkali Aggregate Reaction by Li-A type Zeolite," Proceedings for the 113th Academic Conference of the Society of Inorganic materials 2006 (in Japanese).

[4] Matsumoto T., Miyazaki T. Goto Y., "Synthesis and Characterization of Li-type EDI zeolite," Journal of the European Ceramic Society, Vol.26, pp.455-458, 2006.

[5] Uehara M., Mizuno K., Sato T., Matsumoto T., Goto Y., "Preparation of Li-containing Zeolites from Metakaolin,” Nendo Kagaku, Vol.50, No.1 2011 (in Japanese). 